\title{
Sarcoma de Ewing extraesquelético subcutáneo primario / PNET. Reporte de caso y revisión de la literatura
}

\author{
Primary subcutaneous extraskeletal Ewing's sarcoma (PNET). A case report and literatura review
}

Patricia K. Paucar-Lescano ${ }^{1,2, a, b, c, ~}$ Alex Ventura-León ${ }^{3,4, b, d}$, Alejando Dagnino-Varas 5,d

RESUMEN

La familia de tumores del Sarcoma de Ewing (ES) es un grupo poco habitual de neoplasias malignas que pueden localizarse en regiones: óseas como extraóseas. El tumor neuroectodérmico primitivo (PNET), muestra más diferenciación neuronal que el Sarcoma de Ewing (ES) y rara vez se presenta en piel y tejidos subcutáneos. Ocasionalmente se presenta como un cáncer de piel primario, que tiene como características clínicas: una tumoración superficial, en promedio mide $2-3 \mathrm{~cm}$, de consistencia blanda, móvil y algunas veces dolorosa. Los lugares más afectados son espacios paravertebrales, extremidades inferiores, cabeza, cuello y pelvis. Describimos el caso de un varón de 13 años, con 2 tumores superficiales en cuero cabelludo, el examen histológico presentaba una proliferación de células de pequeño tamaño, basófilas, monomorfas; con perfil inmunohistoquímico FLI1 positivo, compatible con PNET.

PALABRAS CLAVE: Sarcoma de Ewing, piel, tumores neuroectodérmicos periféricos primitivos. (Fuente: DeCS BIREME).

\section{SUMMARY}

Ewing's sarcomas are a less common malignant neoplasms that can be located both in skeletal and extra skeletal areas. The primitive neuroectodermic tumor (PNET) shows more neuronal differentiation than Ewing's sarcoma and rarely invades the skin and soft tissues. This tumor occasionally presents as a primary skin cancer which has distinctive features such as a superficial tumor with a $2-3 \mathrm{~cm}$, soft, mobile and eventually painful. Most affected areas of the body are paravertebral spaces, lower limbs, head, neck and pelvis. We report here the case of a 13 year old male presenting with 2 superficial tumors located on the scalp, the histo pathological examination revealed a proliferation of small, basophilic and monomorphic cells that showed FLI1 positive immunohistochemistry pattern compatible with PNET.

KEYWORDS: Sarcoma, Ewing, skin, neuroectodermal tumors, primitive, peripheral. (Source: MeSH NLM).

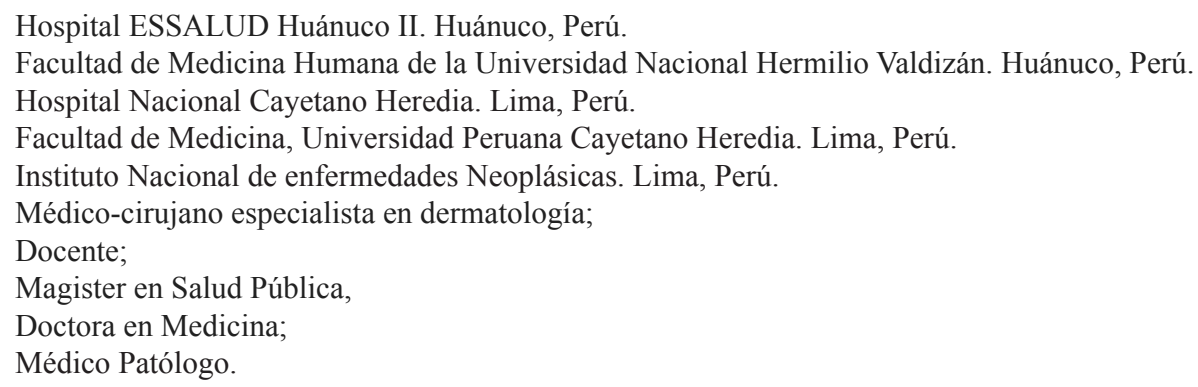




\section{INTRODUCCIÓN}

Elsarcoma deEwing(ES) es un tumoróseo dehuesos largos altamente maligno que se presenta en niños y adultos jóvenes; se han reportado casos de tumores malignos de tejido blando que son indistinguibles de ES y son llamados ES extra esqueléticos (EES); estos tumores son ahora clasificados como familia de tumores ES (ESFT); incluyen ES, EES y tumor neuro ectodérmico primitivo (PNET), que muestra más diferenciación neuronal que ES (1). EES es un tumor de partes blandas raro, agresivo y maligno con alta tasa de recurrencia y que ocurre principalmente en adolescentes y adultos jóvenes entre 10 y 30 años de edad (2). En 1975, Angervall y Enzinger informaron el primer caso de ES extraóseo (3).
ES y PNET forman un solo grupo de tumores del hueso y tejido blando; ES típicamente indiferenciado en un extremo del espectro y PNET con clara evidencia de diferenciación neural en el otro (4).

Se reporta el caso, debido a su rareza y similitud morfológica con otros tumores cutáneos, el PNET puede ser clínicamente y patológicamente subdiagnosticado; se debería incluir en el diagnóstico diferencial, incluso en casos simples, incluyendo estudio anatomo patológico.

\section{PRESENTACIÓN DEL CASO}

Varón de 13 años, mestizo, natural de HuánucoPerú, que acudió a consulta con dos tumores

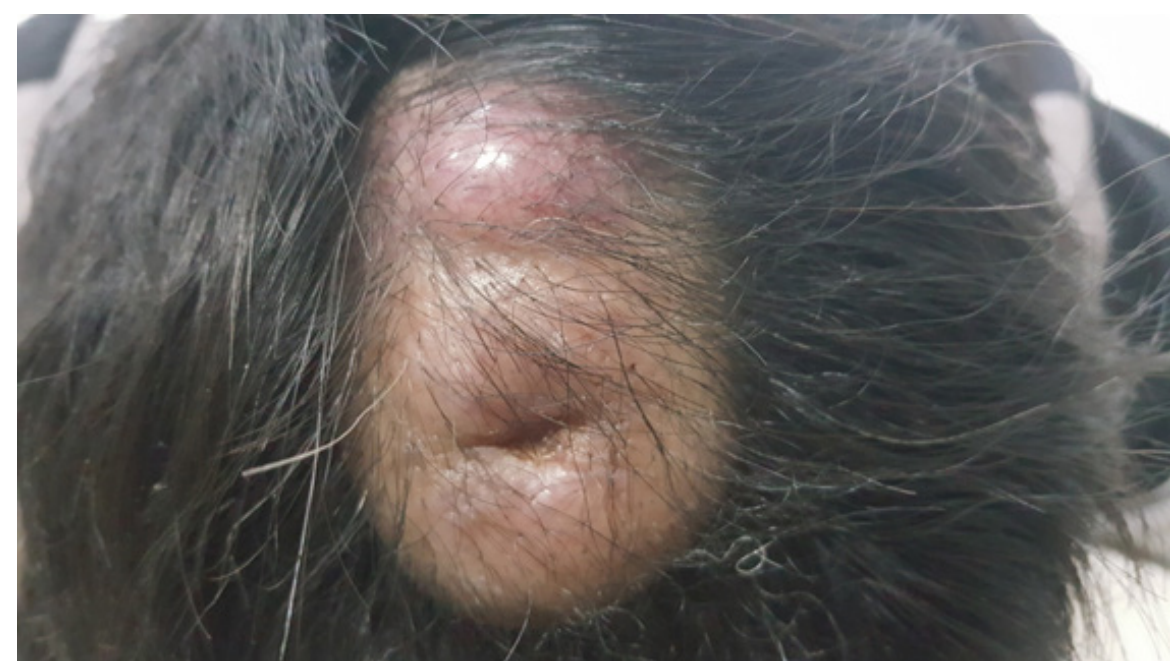

Figura 1. Tumores superficiales en cuero cabelludo; de $3,5 \mathrm{~cm}$ y $2 \mathrm{~cm}$, con rápido aumento de volumen en 4 meses.
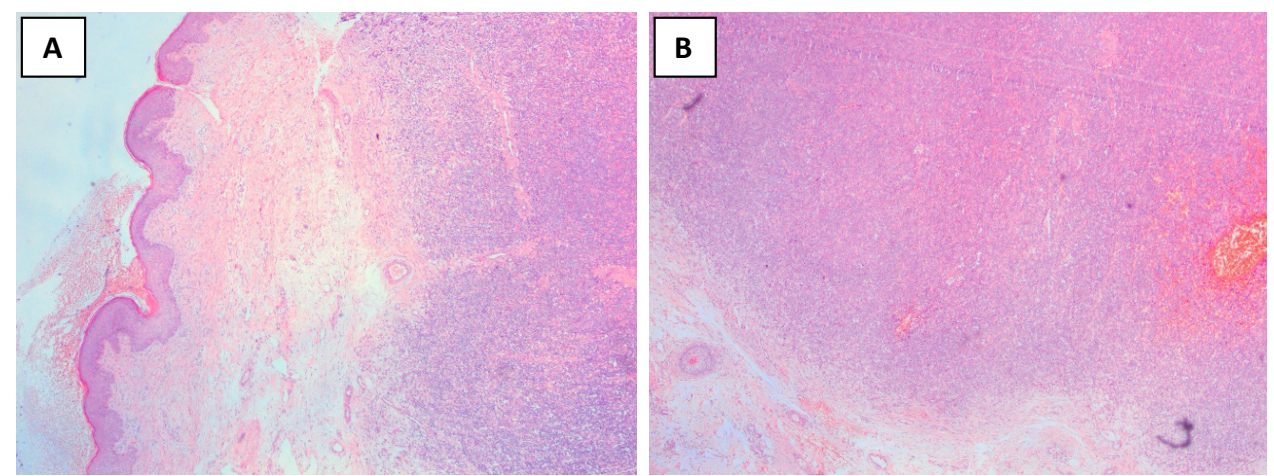

Figura 2. A: 40X, tinción hematoxilina-eosina. Epidermis sin mayores alteraciones y en la dermis presencia de una proliferación de células pequeñas redondas y azules de disposición nodular que ocupa predominantemente la dermis reticular y abarca hasta TCSC. B: 40X, tinción hematoxilina-eosina. En la dermis hay presencia de una proliferación de células pequeñas redondas y azules de disposición nodular que ocupa predominantemente la dermis reticular y abarca hasta TCSC. 
superficiales, ubicados en el vertex: el más grande de $3,5 \mathrm{~cm}$. de diámetro del tono de piel del paciente, tenía una depresión en el centro por haber sido punzada con anterioridad y la segunda de $2 \mathrm{~cm}$. de diámetro, de color eritemato-violáceo, de consistencia gelatinosa, ligeramente móviles y dolorosos a la palpación, con rápido aumento de volumen en cuatro meses (figura $1)$.

Se realizó biopsia por losange del tumor $2 \mathrm{~cm}$ (más pequeño), cuya descripción histológica fue, epidermis sin mayores alteraciones y en dermis presencia de una proliferación de células pequeñas redondas y azules de disposición nodular que ocupa predominantemente la dermis reticular y abarca hasta TCSC (figura 2A y $\mathrm{B}$, figura 3). Se realizó examen inmunohistoquímico FLI1 (friendleukemia virus integration 1), donde se aprecia sobre expresión nuclear de FLI-1 (figura 4).

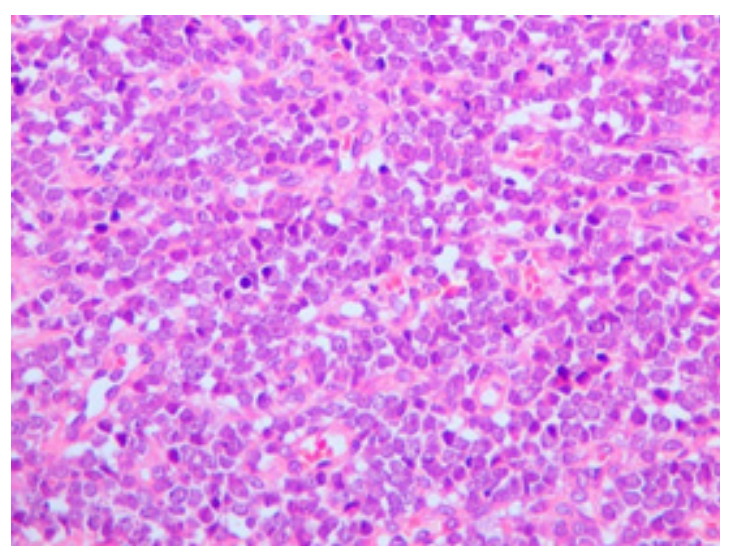

Figura 3. 400X, tinción hematoxilina-eosina. Imagen a mayor aumento de la proliferación celular en donde se observa con mejor detalle el escaso citoplasma y la uniformidad entre ellas.

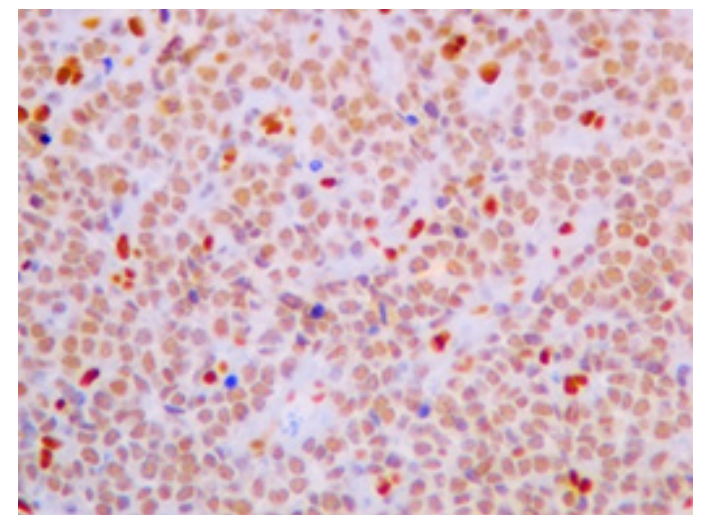

Figura 4. 400X, tinción FLI1 (friendleukemia virus integration 1): Sobre expresión nuclear de FLI-1.

\section{DISCUSIÓN}

La familia de tumores del sarcoma de Ewing (ESFT) representa una familia de neoplasias morfológicamente similares, incluyendo el sarcoma de Ewing clásico (ES) del hueso, el ES extra esquelético, el tumor de células pequeñas de la región toraco-pulmonar (tumor de Askin) y tumores neuro ectodérmicos primitivos del tejido blando (PNET) (5).

El sarcoma de Ewing extraóseo / tumores neuro ectodérmicos periféricos (ESS / PNET), son neoplasias raras que representan aproximadamente de 10 a $15 \%$ de los sarcomas de tejidos blandos en niños y el $5 \%$ de los sarcomas de tejidos blandos en adultos (6). Clínicamente, morfológica y genéticamente ESS/ PNET, comparten muchas características, lo que respalda la hipótesis de que estas dos neoplasias están histogenéticamente relacionadas y son consideradas como parte de la misma familia de tumores (7).

Los sitios extra esqueléticos comúnmente afectados incluyen los espacios paravertebrales, extremidades inferiores, cabeza, cuello y pelvis; otras ubicaciones raras de EES incluyen el retroperitoneo, epiplón, órbita, piel y pared torácica; es más probable que surjan tumores extra esqueléticos desde ubicaciones axiales y es menos probable que aparezcan de la pelvis (8).

El cuadro clínico es poco común., usualmente involucra tejidos subcutáneos o músculos profundos, y rara vez ocurre como cáncer de piel primario; se presenta como una tumoración superficial de $2-3 \mathrm{~cm}$, de consistencia blanda, móvil, a veces dolorosa; el tumor se encuentra en la dermis media o profunda o tejido superficial subcutáneo y puede involucrar a la papila dérmica, con presentación tumoral pedunculada (9).

El diagnóstico diferencial se realiza con otras neoplasias compuestas de células redondas azules pequeñas: neoplasias primarias y metástasis cutáneas; las neoplasias primarias pueden ser: carcinoma de células de Merkel, espiradenoma ecrino, linfomas, sarcoma de células claras, rabdomiosarcoma, tumor rabdoideo maligno, tumor neuro ectodérmico primitivo maligno, carcinoma mioepitelial, histiocitoma fibroso angiomatoide, tumores anexiales pobremente diferenciados y sarcoma granulocítico; y las metástasis cutáneas pueden derivar de: sarcoma de Ewing óseo, carcinoma neuroendocrino de células grandes, carcinoma de pulmón de células pequeñas y neuroblastoma (9). 
El diagnóstico puede requerir varias técnicas auxiliares tales como citología por aspiración, tinciones histoquímicas, inmunohistoquímica, microscopía electrónica, citogenética y genética molecular de traslocaciones; el aspecto histológico de células redondas pequeñas, positivo para CD99 en característico patrón membrana en la histoquímica y la translocación cromosómica específica que implica genes EWSR1 en el cromosoma 22q12 son criterios esenciales para diagnóstico de EES (10).

El tratamiento se realiza inicialmente con resección quirúrgica, asociado o no a la quimioterapia o radioterapia; según tamaño y ubicación del tumor, la radioterapia se usa cuando hay márgenes positivos después de la resección quirúrgica (11). Por lo tanto, para la enfermedad se requiere una estrategia terapéutica multimodal, agresiva (12). En pacientes con enfermedad localizada se estima que la tasa de supervivencia a 5 años es alrededor de $70 \%$, debido al considerable progreso de la terapia tanto local como sistémica, durante las últimas 4 décadas (13).

El carácter menos agresivo del cuadro clínico cutáneo probablemente se produce debido a la ubicación superficial, tumores más pequeños y de fácil acceso, permitiendo la detección a través del examen clínico o por el propio paciente, diagnóstico precoz y resección quirúrgica completa, evitando el inicio de metástasis (10). Cerca de 30-40\% de pacientes con ESS/PNET sufren de tumores recurrentes y tienen un muy mal pronóstico, la supervivencia a 5 años después de la recurrencia de estos pacientes fue de a $10-15 \%$, y de $7 \%$ para aquellos cuya enfermedad reapareció en 2 años (7).

Una gran cohorte de pacientes con sarcoma de Ewing localizado tratado con modernos protocolos de quimioterapia, determinaron que la edad avanzada, la raza no blanca y la VSG basal elevada son factores pronósticos adversos independientes en pacientes con EES, y que el patrón de recaída no difiere entre EES y ES esqueléticos (14).

Antes del uso de la terapia sistémica multiagente, la tasa de supervivencia a largo plazo, con cirugía o radiación era inferior al 10\%; con la adición de la quimioterapia multi- farmacológica para la cirugía o radiación, las tasas de supervivencia a 5 años varían de 49 a $60 \%$ en pacientes con enfermedad localizada, siendo los regímenes de quimioterapia estándar actual: vincristina, doxorrubicina y ciclofosfamida, alternando con ifosfamida y etopósido (régimen VAC-I / E) para la enfermedad localizada, y vincristina, doxorrubicina y ciclofosfamida (régimen VAC) en la enfermedad metastásica (15).

Aunque son tumores raros, los EES/PNET, se deben considerar en el diagnóstico diferencial, una adecuada evaluación clínica, estudios histológicos, inmunohistoquímicos y citogenéticos, realizados a tiempo y un tratamiento apropiado resultan fundamentales para su correcto manejo, debido a que la enfermedad cutánea, generalmente, presenta un curso lento y un pronóstico favorable.

\section{Declaración de financiamiento y de conflictos de interés:}

El reporte fue financiado por los autores; declaran no tener conflictos de interés.

\section{Contribución de autoría:}

PPL: Contribución concepción y diseño del artículo, redacción del artículo, revisión crítica del artículo, aprobación de la versión final, aporte de pacientes y material del artículo. AVL: Contribución en estudio histopatológico y colaboración en la revisión crítica del artículo. ADV: Contribución de los estudios complementarios inmunohistoquímicos y ampliación molecular, así como colaboración en la revisión crítica del artículo.

\section{Correspondencia:}

Patricia Karen Paucar Lescano

Jr. Bolivar 325 departamento 801.Huánuco, Huánuco, Perú

Teléfono: 51980059325

Correo electrónico: drakarenpaucar@gmail.com

\section{REFERENCIAS BIBLIOGRÁFICAS}

1. Isefuku S, Seki M, Tajino T, et al. Ewing's sarcoma in thespinalnerveroot: A case report and review of the literature. Tohoku J Exp Med. 2006; 209:369-77. DOI: https://doi.org/10.1620/tjem.209.369

2. Shin J, Lee H, Rhim S, Cho K, Choi C, Suh D. Spinal epidural extraskeletal Ewing sarcoma: MR findings in two cases. AJNR. 2001; 22:795-8. (Citado el 14 de noviembre del 2017) Disponible en: http://www.ajnr. org/content $/ 22 / 4 / 795$

3. Basma E, Hajar H, Nabil M, Anis B, Samir B. Breast Ewing sarcoma / primitive neuroectodermal tumor: A case report and a review of the literature. Breast. 2012; 3:3792. (Citado el 14 de noviembre del 2017) 
Disponible en: http://www.webmedcentral.com/ wmcpdf/Article_WMC003792.pdf

4. Srivastava S, Arora J, Parakh A, Goel R. Primary extraskeletal Ewing's sarcoma/ primitive neuroectodermal tumor of breast. IJRI. 2016; 26(2):226-30. doi: 10.4103/0971-3026.184408

5. Ross K, Smyth N, Murawski C, Kennedy J. The Biology of Ewing Sarcoma. ISRN Oncology. 2013; 759725:1-7. Doi: http://dx.doi.org/10.1155/2013/ 759725

6. Mardekian S, Gandhe A, Miettinen M, Pack S, Curtis M, Abdullaev Z. Two cases of spinal, extraosseous, intradural Ewing's sarcoma/peripheral neuroectodermal tumor: radiologic, pathologic, and molecular analysis. J Clin Imaging Sci. 2014; 4:6 doi: $10.4103 / 2156-7514.126050$

7. Grassetti L, Torresetti M, Brancorsini D, Rubini C, Lazzeri D, Di Benedetto G. A peculiar case of large primary cutaneous Ewing's sarcoma of the foot: Case report and review of the literature International. Int J Surg Case Rep. 2015; 15:89-92. doi: 10.1016/j. ijscr.2015.08.024

8. Galyfos G, Karantzikos G, Kavouras N, Sianou A, Palogos K, Filis K. Extraosseous Ewing Sarcoma: Diagnosis, prognosis and optimal management. Indian J Surg. 2016; 78(1):49-53. doi: 10.1007/ s12262-015-1399-0

9. Oliveira Filho Jd, Tebet AC, Oliveira AR, Nasser K. Primary cutaneous Ewing sarcoma-case report. An Bras Dermatol. 2014; 89(3):501-3.
10. Chow E, Merchant TE, Pappo A, Jenkins JJ, Shah AB, Kun LE. Cutaneous and subcutaneous Ewing's sarcoma: an indolent disease. Int J Radiat Oncol Biol Phys. 2000; 46(2):433-8.

11. Ellison DA, Parham DM, Bridge J, Beckwith JB. Immunohistochemistry of primary malignant neuroepithelial tumors of the kidney: a potential source of confusion? A study of 30 cases from the National Wilms Tumor Study Pathology Center. Hum Pathol. 2007; 38(2):205-11.

12. Halefoglu A. Presentación de un sarcoma extraóseo de Ewing como una masa mediastínica posterior. Arch Bronconeumol 2013; 49:82-4. DOI: 10.1016/j. arbres.2012.02.020

13. Geens L, Van Robays J, Geert V, Van der Speeten K.An Unusual Location of Extraosseous Ewing's Sarcoma. Case Rep Oncol. 2013; 6(2):293-302. doi: $10.1159 / 000351836$

14. Cash T, McIlvaine E, Krailo M, et al. Comparison of clinical features and outcomes in patients with extraskeletal versus skeletal localized Ewing sarcoma: A report from the Children's Oncology Group. Pediatr Blood Cancer. 2016; 63(10):1771-9. doi: 10.1002/ pbc. 26096

15. Valdes M, Nicholas G, VermaS, Asmis T. Systemic Therapy Outcomes in Adult Patients with Ewing Sarcoma Family of Tumors. Case Rep Oncol. 2017; 10(2):462-472. doi: 10.1159/000475806

Recibido: 20/05/2019

Aceptado: 30/12/2019 